\title{
Ein Brief Fichtes über sein Verhältnis zur Kantischen Philosophie.)
}

\author{
(Hamb. Mscr. IV, p. 50.)
}

Mitgeteilt von M. Grunwald in Hamburg.

(Abschrift von Villers' Hand.)

Brief von Ficlite an d. Hn. Appia.

Berlin, d. 23. Jun. 1804.

Sie erhalten hier, mein werthgeschäzter Herr und Frennd, einen Begriff von der Transcendental-Plilosophie nnd von Kant und von meinen Bemuhungen um dieselbe, so scharf bestimmt, als man iln geben kann, olne in die Sache selbst einzuweihen. -

Damit nicht auch dieser Begriff völlig leer, und unverstanden bleibe, dazı gehört:

1) in Beziehung auf $\S 2$, dass man sich in das Bewusstseyn, durch cine gar nicht gewöhnliche Anstrengung der Aufmerksamkeit, recht

1) Dieser Brief ist, soweit sich wenigstens bis jotzt feststellen lüsst, noch unged ruckt. Sollte er doch irgendwo - vielleicht an einem weniger bekannten Orte - schon abgedruckt sein (woriiber wir den ev. Finder un freundliche Mitteilung bitten), so wiire der Neudruck nichtsdestoweniger eben darum zweckmissig.

Wo das Original des Bricfes sich befindet, ist ebenfalls nicht z11 eruieren gewesen. Viclleicht in irgend einer Autographensamulung? Auch hieriiber bitten wir un eventuelle Mitteilung.

Diese Bitte un freundliche Mitwirkung unscrer Leser erstreckt drittens sich anf die uns unbekannte Person des Adressaten, des Herrn Appia. Vielleicht war dersclbe ein Schweizer: in Genf wenigstens kommt derselbe Name noch hente vor.

Die Abschrift des Briefes befindet sich in dem auf der Hamburger Bibliotck aufbewahrten Nachlass von Cbarles de Villers. Eine "Auswahl aus dem handschriftlichen Nachlass" desselben hat schon M. Isler herausgegeben, u. d. T. „Briefe von L. Constant-Gürres - Güthe" u. s. w. (Hamburg, Meissner 1879). Eine Nachlese gab ich selbst heraus u. d. 'I. "Briefe ans dem Kantkreise", in der Altpreuss. Monatschr. XVII, S. 2×6-2!9 (1581)).

II. $\mathrm{V}$. 
lebendig hineinversetze. In der gewöhnlichen Reflexion, die ich eine verblasste nenne, wird das Bewusstseyn sogleich wieder objektivirt, und so uns entfremdet, und da entsteht denn der $\S 1$ gerügte täuschende Schein unseres Geistes, Seele und welcherlei Namen dieses Gespenst noch haben mag. Und auf diesem Wege gelangt man nie auch nur zur Ahndung des transcendentalen Organismus. Man muss lebendig inne werden, dass man im Bew usstseyen eben unmittelbar Selber das Bewusstseyen sey, und nicht mehr, noch minder.

2) in Beziehung auf $\$ 4$ und die dort sogar von $K$ ant gerügte Empirie, - Man muss wenigstens, vorläufig als möglich annehmen, dass diejenige Weisheit, in der wir alle aufgewachsen: dass nehmlich Erfahrung, Beobachtung, Empirie doch dass höchste und letzte bleibe, und dass darüber nie jemand herauskommen werde, - dass, sage ich, diese Weisheit doch wohl die rechte wahre, eigentliche 'Thorheit seyn dürfte; denn dies gerade ists, was (ausser den Alten insgesammt, z. B. Plato, Jesus, und die ganze Christenheit) Kant (ohnerachtet dieser eben nicht bis zn Ende sich selber gleich geblieben) und i ch, voraussetzen. Jemandem den Beweiss führen, dass man schlechthin a priori wissen könne, und dass nur durch das a priori Ordnung und Evidenz in unser Wissen komme, können wir nur durch die That selber, indem wir ihn selbst wirklich zu diesem Wissen a priori über alle Erfahrung hinaus erheben. Für diesen Zweck muss er aber zu allererst in unsere Voraussetzung hineingehen. -

An diese beyden unerlässlichen Bedingungen stösst es sich nun sogar sehr häufig in Deutschland, und es giebt auch bey uns der Subjekte, welche nur das, was ich in der Beilage niedergeschrieben, wüssten, oder zu begreifen fähig wären, weit weniger als etwa die Ausländer glauben. Sollten nun, wie man ohne von Patriotismus verblendet zu seyn, wohl annehmen kann, in der Regel nehmlich, und im allgemeinen Durchschnitt, die übrigen Nationen des neuern Europa in ihren Geistesoperationen, noch weit verblasster, zerstrenter und flacher, und in die Empirie noch weit tiefer versunken seyn, als Selber die Dentschen, so lässt bei ihnen für ein System wie das beschriebene, sich noch weit weniger Glück hoffen. Sie werden daher, wertgesch. H. und Frd., eine scharfe Auswahl zu treffen haben unter denen, denen Sie sich über diesen Gegenstand mittheilen, and sich gefasst halten müssen auf die sonderbarsten Urtheile.

Fichte.

Aphorismen über das Wesen der Philosophie als Wissenschaft.

$\S 1$. Alle Philosophie bis auf Kant, batte zu ihrem Gegenstande das Seyn (objectum, ens) - (im Dualismus z. B. wurde das Bewasstseyn selber, als bewusster Geist, Seele, u. s. f. zum Seyn). Der Zweck dieser Philosophie war, den Zusammenhang der mannigfaltigen Bestimmungen dieses Seyns zu begreifen.

$\S 2$. Alle ubersahen lediglich aus Mangel an Aufmerksankeit, dass kein Seyn, ausser in einem Bewusstseyn und umgekehrt, kein Bewusstseyn ausser an einem Seyn, vorkomme; dass daher das eigentliche Ansich, als 
Objekt der Philosophie weder Seyn, wic in aller vorkantischen Philosophic noch Bewusstseyn [8ei], wie freylich nicht einmal versucht worden; sondern Soyn + Bewusstseyn, oder Bew. + Sein $=$ der absoluten Einheit beyder, jenseit ihrer Geschiedenheit, seyn mulsse. Kant war es, der diese grosse Entdecknng machte, und dadurch Urheber der TranscendentalPhilosophie wurde.

Corollarium. Dass daher jene wunderbare Fragen, wie das Seyn ins Bernsstseyn, oder das Bewusstsein zum Seyn komme, welche durch Influxus physicus, Systema causarum occasionalium, Harmonia praestabilita [beantwortet werden sollten], gelöst, indem Seyn and Bewusstseyn ursprünglich ja nicht getrennt sind, drum auch nicht vereinigt werden können, sondern an sich Fins und dasselbe sind. -

§ 3. Zusatz. Es versteht sich, dass auch nach dieser totalen Umänderung des eigentlichen Objekts, die Philosophie noch immer ihre alte Aufgabe behalte, den Zusammenhang der mannigfaltigen Bcstimmangen jenes Grundobjekts begreiflich za machen.

$\$ 4$. In diesem letztern Geschäfte der Ableitung kann man nun

Entweder also verfahren, dass man gewisse Grandunterschiede, welche nur in empirischer Selbstbeobachtung gefunden seyn können, als nicht weiter zu vereinigend, voransetze, and anf jede dieser besondern Grund-Einheiten nun das aus jeder abzuleitende zurückführe; welches theils eine unvollständige, in sich selber nicht zum Ende, d.h. zar absolaten Einheit, gekommene, theils eine zum Theil auf empirische Data gegründete, drum nicht streng wissenschaftliche, Philosophie geben würde, die doch (wegen $\S 2$ ) [eine] transcendentale bleibt.

Eine solche Philosophie ist die Kantische. -

$\S 5$. Oder man kann also verfahren, dass man jene ursprüngliche Einheit des Seyns and Bewasstseyns $(\S 2)$ in dem, was sie an sich, und unabhängig von ihrer Spaltung in Seyn und Bewusstseyn, ist, durchdringe und darstelle. - (Ich nenne diese Einheit Vernunft, o 2oyos, ut in Evangelio Joannis, Wis s e n, ja nicht zu verwechseln mit Bewusstseyn, was ein tieferes, nur dem Seyn gegenüber befindliches Disjunctionsglied

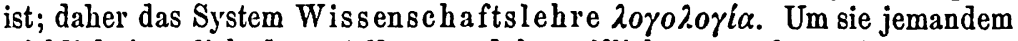
wirklich innerlich darzustellen, und begreiflich $\mathrm{zu}$ machen, dazu gehört eine lange Vorbereitung desselben durch die abstrakteste Spekulation). Wird man sie, jene Einheit, recht dargestellt haben, so wird man zugleich den Grund, warum sie in Seyn und Bewusstseyn sich spalte, einsehen, [man wird] einsehen, warum es in dieser Gespaltenheit auf eine bestimmte Weise sich weiter spalte: alles schlechthin a priori, ohne alle Beihülfe empirischer Wahrnehmung, auf jener Einsicht der Einheit; und also wahrhaftig das All in dem Einen, und das Eine im Allen begreifen; welches von jeher die Aufgabe der Philosophie gewesen. Diese jetat beschriebene Philosophie ist die

Wissenschaftslehre.

Erläuternder Zusatz.

Was die letztere weitere Spaltung des schon als Eins begriffenen Seyns nnd Bewusstseyns betrifft, so findet sich in der Wissenschaftslehre, 
dass diese geschehe, zufolge des Bewusstseyns und nach seinen immanenten Gesetzen; dass daher das Seyn, an und für sich, und abgetrennt gedacht vom Bewasstseyn, durchaus Eines sey, so wio die Vernunft selber, und dass es nur in seiner Vereinigung mit dem Bewusstseyn sich spalte, weil das letztere, zufolge seines eignen Wesens, sich nothwendig spaltet; mithin nur im Bewusstseyn es ein mannigfaltiges Seyn giebt; z. B. (so nehmlich findet es sich in der Wissen[schafts]l[ehre]) spaltet [dieses] sich zuförderst in ein sinnliches und übersinnliches Bewusstseyn, was auf das Seyn angewendet, ein sinnliches und übersinnliches Seyn geben muss. Das Uebersinnliche spaltet sich hinwiederum, nach einem hier nicht auszuführenden Gesetz, in religiöses und moralisches Bewusstseyn, was auf das Seyn angewendet, einen Gott giebt, und ein sittliches Gesetz; das Sinnliche spaltet sich wiederum in ein sociales und in ein Natur-Bewusstseyn, was auf das Seyn angewendet, ein Rechtsgesetz und eine Natur giebt. Endlich wird, eben als Resultat der absoluten, (d. $h$. unendlichen, nie $z \mathbf{n}$ erfüllenden) Spaltung im Bewusstseyn, das absolut gespaltene Seyn, d. h. die Natur, ausgedehnt durch einen unendlichen Raum, das Bewusstseyn ausgedehnt durch eine unendliche Zeit; welche Zeit aber, und Raum, ebenso wenig, als die ersterwähnten Disjunctionen, an sich, d. h. im reinen Seyn, oder auch in der reinen Vernunft, sondern nur im Bewusstseyn, stattfinden. Demnach ist das Bewusstseyn mit diesen seinen Gesetzen und Resultaten keineswegs Täuschung, denn es ist kein Seyn, und es ist keine Vernunft, ausser im Bewasstseyn. Aus demselben Grunde können wir das Bewusstseyn, und seine nothwendigen Resultate im Leben nie aufgeben; ohnerachtet wir wissen können und sollen, dass diese Resultate nicht an sich gültig sind, um uns dadurch von den ungeheurn Verwirrungen und Widersprüchen, welche durch jene falsche Voraussetzung von jeher angerichtet worden, $\mathrm{zu}$ verwahren.

Berlin, d. 23. Jun. 1804.

Fichte. 